\title{
Study of analysis method of interior noise in railway cars by means of ray tracing method
}

\author{
Takashi YOSHIZAWA*, Toshihiko MOCHIDA** and Toru YAMAZAKI*** \\ ${ }^{*}$ Research \& Development Group, Hitachi, Ltd. \\ 832-2 Horiguchi, Hitachinaka, Ibaraki 312-0034, Japan \\ E-mail: takashi.yoshizawa.cc@hitachi.com \\ ${ }^{* *}$ Railway Systems Business Unit, Hitachi, Ltd. \\ 794 Higashitoyoi, Kudamatsu, Yamaguchi 744-8601, Japan \\ ***Department of Mechanical Engineering, Kanagawa University \\ 3-27-1 Rokkakubashi, Kanagawa-ku, Yokohama, Kanagawa 221-8686, Japan
}

Received: 15 October 2018; Revised: 23 March 2019; Accepted: 2 August 2019

\begin{abstract}
We have studied the analysis method of interior noise in railway cars by means of a ray tracing method. Firstly, by using a point source as input, sound distribution inside a car was analysed and compared with the measurement result obtained by a loud speaker test. It was found that the analysis result of sound decay along the longitudinal direction of the car did not always match the measurement result. It is possibly because the wave character of the sound cannot be strictly simulated by the ray tracing method. However, for the purpose of analysing the sound distribution while the train is running, it may not be necessary to accurately simulate the sound decay in the longitudinal direction, because the sound sources are distributed everywhere inside the running car and therefore the importance of the sound decay along the longitudinal direction may be low. Based on this perspective, we have proposed the interior noise analysis method for the running condition by applying multiple sources inside the car. The amplitude of each source was estimated from the measured vibration of the interior panels as well as from the estimated radiation efficiency of each panel. Validation of the analysis was conducted by comparison with the measured data of the interior noise inside the running train, and it was confirmed that the level of interior noise reduction by the installation of passenger seats matched well between the analysis and the measurement.
\end{abstract}

Keywords : Ray tracing method, Statistical energy analysis, Interior noise, Railway car, Sound distribution

\section{Introduction}

In order to predict the interior noise level of railway cars, statistical energy analysis (SEA) has been widely used to develop a whole vehicle model (Muto et al., 2003), (Poisson et al., 2009), (Yoshizawa et al., 2013). SEA has the advantage that the degree of freedom of the model is much smaller than the finite element method (FEM) or the boundary element method (BEM). Therefore it is suitable for analysing the interior noise of big structures such as railway cars in practical computation time. However, because SEA is a method for analysing the average sound pressure level of the acoustic cavities, it is generally not possible to analyse the sound distribution inside the car in detail, which is affected by the reflection at interior panels, or by the local absorption at objects such as seats. Although FEM or BEM is capable of analysing the sound distribution in detail, the computation time increases rapidly as the number of elements increases. So it is not practical to apply FEM or BEM to the analysis of interior noise in railway cars under current computation ability. On the other hand, in the field of architectural acoustics it is known that the ray tracing method can be used for the analysis of sound propagation in a vast space such as a concert hall. Therefore, applying the ray tracing method to the analysis of interior noise in railway cars was studied here.

The ray tracing method is categorized in geometrical acoustics, which computes the sound reflection at a wall by considering a number of rays. As opposed to wave equation based methods such as FEM or BEM, the ray tracing 
method cannot accurately simulate wave characters such as diffraction, diffusion, or interference. Therefore, it is difficult to obtain strictly accurate results with the ray tracing method, especially in low frequencies, in which the wave character of the sound is more prominent than in high frequencies. However, the ray tracing method has the advantage that the computation time is very short compared with FEM or BEM, and the effect of diffraction, diffusion, or interference can be taken into account to some extent with approximative corrections. In this sense, it may be possible that the ray tracing method can be applied to the analysis of the sound distribution caused by the shape of the interior space and/or the local absorption at the seats, with reasonable accuracy and practical computation time. Therefore, we have decided to apply the ray tracing method for the analysis of interior noise in railway cars.

The use of the ray tracing method for interior noise analysis in railway vehicles has been studied in past literature. Gabet developed a ray-tracing model and validated the analysis results with a full scale vehicle test (Gabet et al., 2008). The validation results seem satisfactory, but their model did not include internal fittings such as seats, so the effect of seats on the noise distribution inside the vehicle is not clear. Forssen conducted a scale model measurement and compared the results with those obtained with the SEA model as well as the ray-tracing model (Forssen et al., 2012). Their model included the seats inside the vehicle, but the results were compared only at the aisle position, so it is unknown how valid the analysis results are at the seat location, where the SPL is assumed to be more affected by the diffracted sound at the edge of the seats.

In this paper, the ray tracing model for the full scale car is developed both with and without the passenger seats. The validation of the model is conducted in comparison to the measurement by a loud speaker test, and the influence of the seats on the accuracy of the model is discussed. Also, in order to analyse the interior noise while the train is running, in which a number of sources are usually distributed inside the car, multiple sources are placed on the internal surface of the car. Then, the allocation method of input power to each source is presented, based on the measured vibration data of the interior panels in running condition. The validity of the applied multiple sources is checked against the measured sound distribution inside the car when the seats are fitted. Finally, the accuracy of the simulation model in comparison to the measurement is verified by the sound change due to the installation of the seats.

\section{Overview of the ray tracing method}

The ray tracing method is one of the geometrical acoustic modelling techniques (Savioja et al., 2015). It assumes a number of rays that are emitted from a sound source and traces the path of each ray whenever they are reflected at the surface of the boundary until a certain number of reflections are counted or the energy of each ray is attenuated to a certain level. Attenuation of the ray energy is calculated according to the absorption of the surface where the ray is reflected or the absorption of the air in which the rays are traveling. The sound pressure level (SPL) at a certain position is calculated by the number of rays that pass through a virtual sphere of a certain diameter assumed at the receiver position. Figure 1 illustrates the basic concept of the ray tracing method. Reflection of the rays is assumed to be specular reflection at each surface (i.e. the incidence angle and the reflection angle is the same), and the wave phenomenon at the boundary such as diffraction or diffusion is neglected by the basic ray tracing concept (Architectural Institute of Japan, 2012). However, the ray tracing software used in this study can take into account approximately the first order diffraction based on Kurze-Anderson's formula (LMS, 1998) as shown in Equations (1) and (2), where $\Delta L_{B}$ is the attenuation in $\mathrm{dB}$ due to the screen obstruction, $N$ is the Fresnel number, $\lambda$ is the wave length, and $a, b, d$ is the distance between the source (S), receiver (R), and the diffraction point (D) as shown in Figure 2.

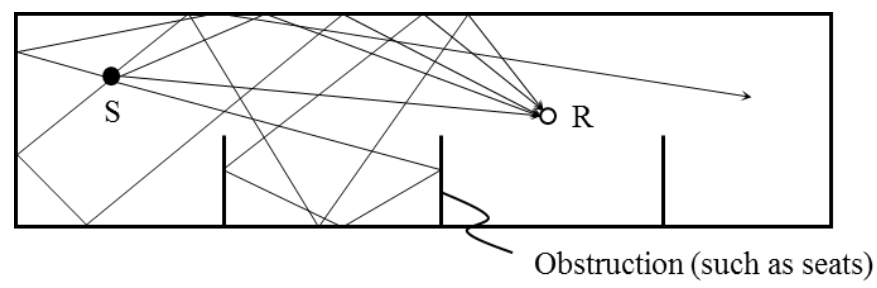

S: Source

R: Receiver

Fig. 1 Basic concept of ray tracing method

$$
\Delta L_{B}=5+20 \log \frac{\sqrt{2 \pi N}}{\tanh \sqrt{2 \pi N}}
$$




$$
N=2\left(\frac{a+b-d}{\lambda}\right)
$$
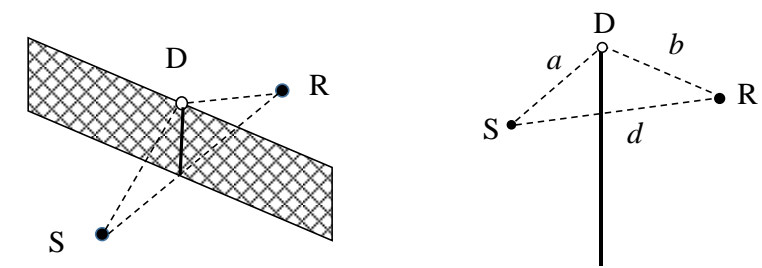

S: Source

R: Receiver

D: Diffraction point

$a, b, d:$ Distance

Fig. 2 First order diffraction effect taken into account in software used (LMS, 1998)

Here, assume a single point source is placed inside a vehicle fitted with seats, whose surface has a certain level of absorption as illustrated in Figure 3. At the position where the source cannot be seen directly and at the same time far from the source, the SPL is more affected by the diffracted sound at the edge of the seats because the reflected sound is gradually attenuated by the absorptive surfaces as the distance from the source becomes longer. However, the ray tracing method can only take into account the first order diffraction, so the diffracted then reflected sound is neglected. Therefore, it is anticipated that the analysis results at a position far from the source are less accurate.

On the other hand, if there are multiple sources inside the vehicle, as is often the case for actual operational trains, the sound propagation from the nearest sources is most important. In particular, if there are multiple sources that are as dense as every two seats or more as shown in Figure 4, the sound propagation from the nearest sources, no matter where the receiver is, can reach the receiver either by direct sound, reflected sound, or the first order diffracted sound, which can all be taken into account by the ray tracing method used in this study. Therefore, there is a possibility that the analysis results for an actual operational train could be more accurate than in the case of only a single source being placed.

In this paper, the validation of the ray tracing model with a single source is conducted by a loud speaker test. Also, the validation with multiple sources for the analysis of the vehicle in a running condition is shown, and the influence of the seats on the accuracy of the model is discussed for both cases.

It should be noted that the diffraction effect at acoustically soft edges such as seat surfaces may be different from that at rigid wall, which is the assumption for Equations (1), (2), and Figure 2. In addition, at the edge of the seat, not only the diffraction but also the transmission of sound could be occurring. However, it is difficult to model such kind of phenomena with current ray tracing method. The purpose of this study is to verify the applicability of the ray tracing method for practical use in spite of those phenomena that cannot be taken into account.

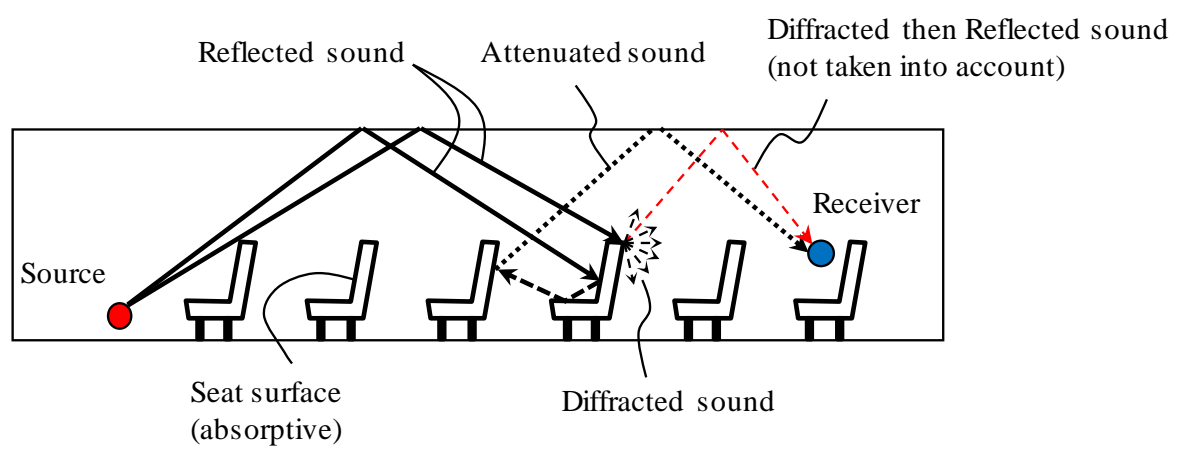

Fig. 3 Schematic of sound propagation when a single point source is placed

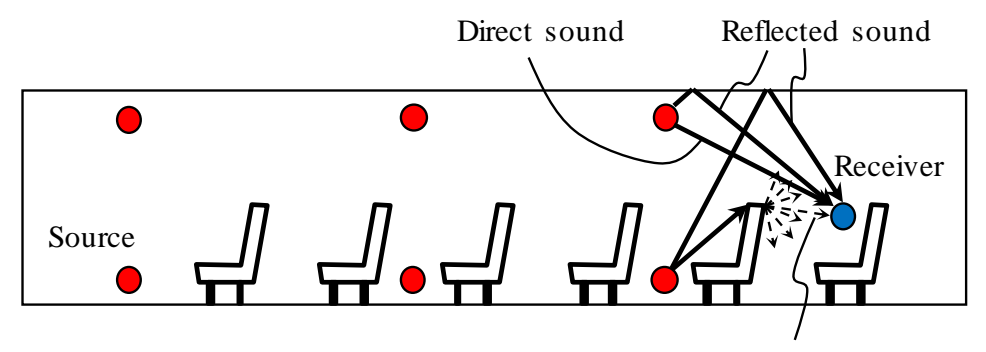

Diffracted sound

Fig. 4 Schematic of sound propagation when multiple sources exist at every two seats 


\section{Sound distribution analysis by using a point source as input \\ 3.1 Analysis model}

Firstly, in order to compare with the loud speaker test done in the real car and to validate the simulation model, analysis by using a point source as input was conducted. Figure 5 shows the analysis model. Interior panels, floor, seats, tables, windows, etc. were modelled as the reflection surfaces of the rays. The absorption coefficient was set on the surface of interior panels, floor, and seats. Other surfaces were considered as complete reflection. As for the interior panels, the absorption coefficient was identified from the reverberation time inside the car, which was measured before the seats and tables were installed. The absorption coefficient of the floor was defined on the basis of that of the carpet laid on the floor. Regarding the seats, the absorption coefficient was calculated from the measurement results of the unit absorption power of a similar seat.

Also, in order to approximately consider the effect of the diffraction at seats, tables, and luggage racks, diffraction lines were set at the edges of them. Only the first order diffraction is considered in the ray tracing software used in this study (LMS, 1998). A point source was placed above one of the bogies at the height of 100mm from the floor in order to simulate the loud speaker test done in the actual car. The measurement was firstly done before the seats and tables were installed in the car, then secondly, after they were installed. Simulation for these two conditions was conducted and will be compared with the measurement in the following section. In regard to the calculation parameters for the ray tracing method, 50,000 rays were used for the analysis, and the calculation was continued until the reflection order of 30 was reached or the dynamic range (ray attenuation) of $90 \mathrm{~dB}$ was fulfilled. Validation of the reverberation time inside the car was also conducted against the measurement results, and good agreement was confirmed for both of the two conditions (with \& without seats and tables), although it is not shown here.

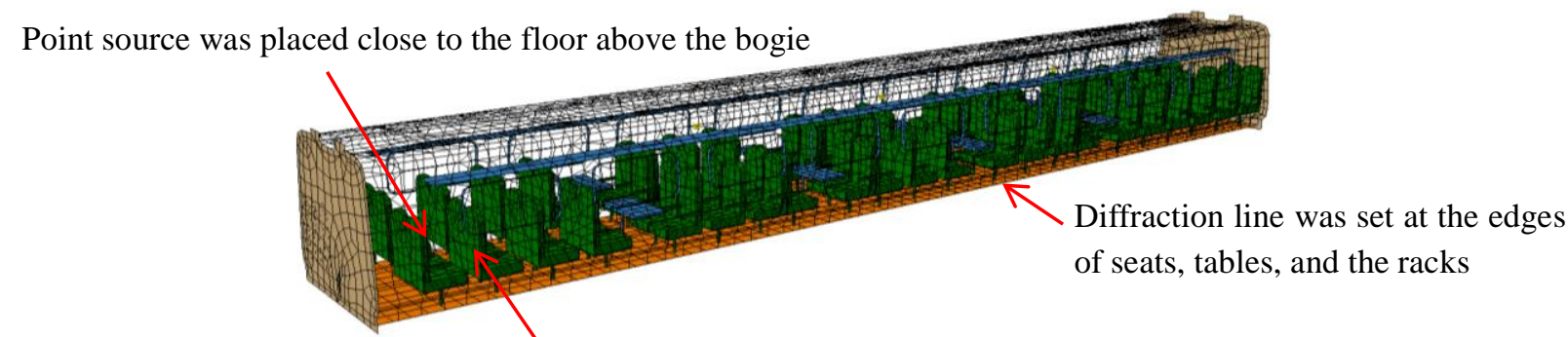

Absorption coefficient was set on the surface of interior panels, floors, and seats

Fig. 5 Analysis model of interior noise by ray tracing method

\subsection{Analysis results without seats and tables}

Firstly, considering the case without seats and tables, the sound distribution in the longitudinal direction was compared between the analysis and measurement as shown in Figure 6. During the measurement, pink noise was generated from the loud speaker, which was placed above the bogie on one side, and the sound was measured at the position away from the speaker (shown as M2, M3, and M4) at the height of $1.6 \mathrm{~m}$ from the floor. Each graph in Figure 6 shows the comparison at these microphone positions in each octave band from $125 \mathrm{~Hz}$ to $2 \mathrm{kHz}$. Because the sound source strength of the speaker during the measurement was unknown, the input strength for the analysis was defined so that the average sound pressure level at these three microphone positions agrees with the measurement. From Figure 6 , it can be seen that the analysis results agree well with the measurement in high frequencies above the $500 \mathrm{~Hz}$ octave band.

On the other hand, in low frequency ranges at the $125 \mathrm{~Hz}$ and $250 \mathrm{~Hz}$ bands, it is observed that the analysed sound decay rate from M2 to M4 is lower than that in the measurement. It was considered that the reason for this difference may derive from the wave character of the sound, which becomes prominent in lower frequencies and cannot be simulated accurately by the ray tracing method. More specifically, in the lower frequency ranges, the phase difference between the sound that directly reaches the microphone and the sound that is reflected at the wall plays an important role because the in-phase and out-of-phase sound from multiple paths are mixed and determine the overall sound pressure level. The primitive ray tracing method is energy based and cannot consider this kind of phase interference, but the ray tracing software used in this study provides the "coherent source option" to calculate the path length difference of each ray and is able to consider the phase interference (LMS, 1998). 

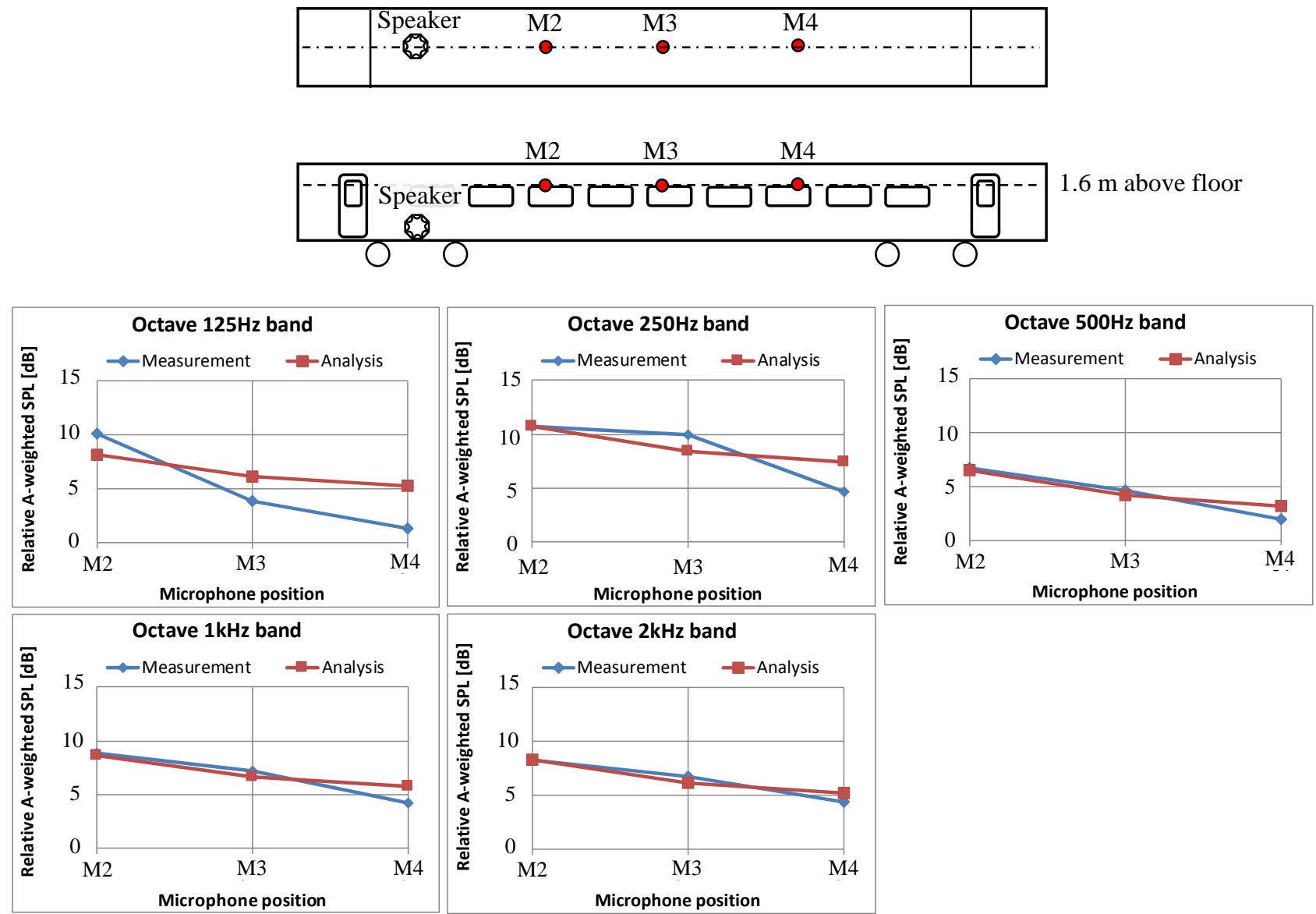

Fig. 6 Analysis results of noise distribution without seats and tables
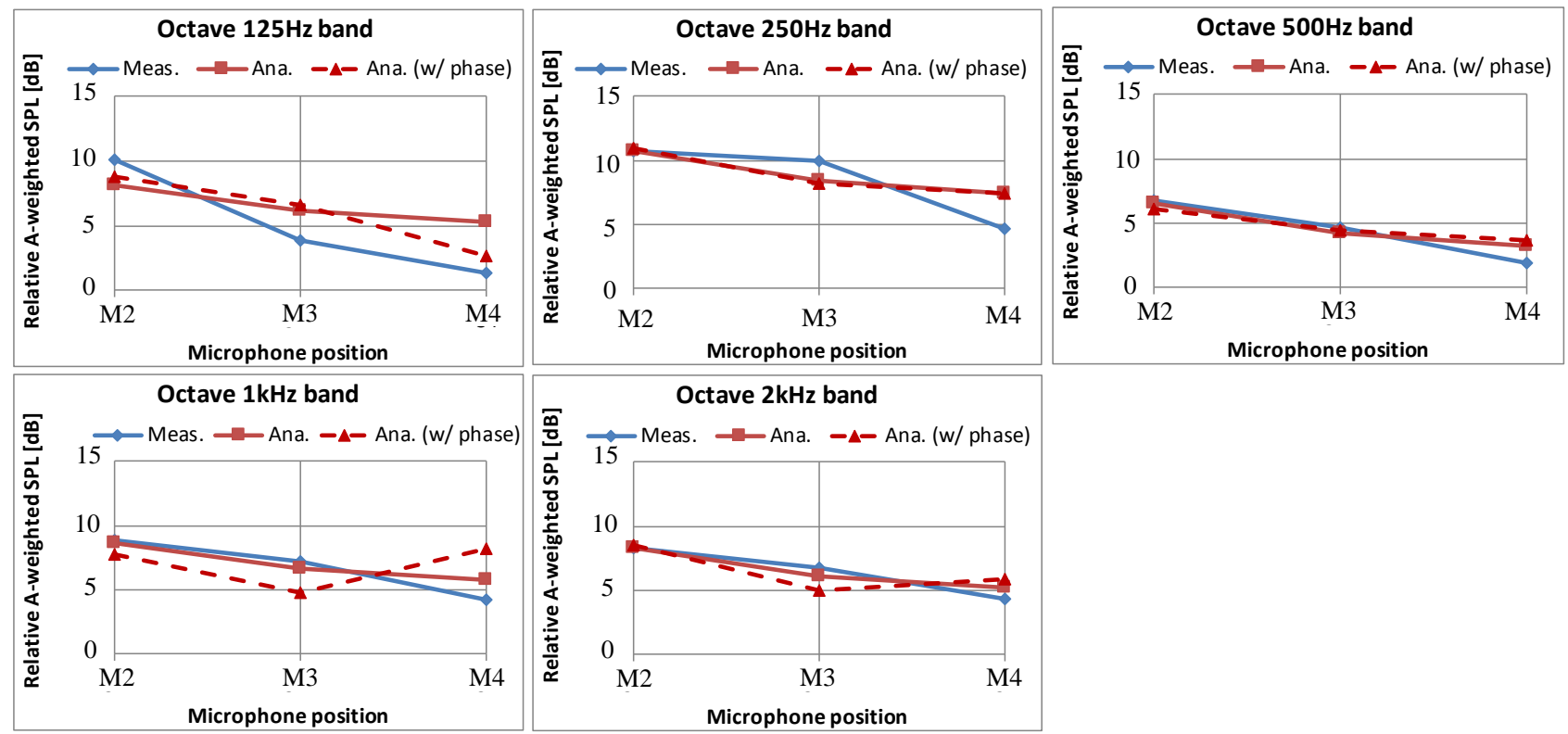

Fig. 7 Analysis results with phase correction (coherent source option) 
In order to examine this phase effect that is important in low frequency ranges, the coherent source option was enabled in the analysis settings. The analysis results with this option are shown in Figure 7 by the dotted line. As can be seen in Figure 7, the octave $125 \mathrm{~Hz}$ result was improved (sound decay from M2 to M4 became closer to the measurement) with the coherent source option, whereas the octave $1 \mathrm{kHz}$ and $2 \mathrm{kHz}$ results were worsened (M4 became larger than M3 as opposed to the measurement).

This can be explained by the difference of the wave length in relation to the path length. In high frequency, because the wave length is much shorter than the path length, the peaks and troughs of the sound pressure caused by the phase interference of the direct sound and reflected sound will occur in short spacing numerically by the ray tracing analysis with the coherent source option. Therefore, the calculation result could be largely affected by a slight difference of the evaluation position. In addition, the SPL is calculated at discrete frequency by logarithmic step with the ratio of 1.05 (e.g. $100 \mathrm{~Hz}, 105 \mathrm{~Hz}, \ldots, 1 \mathrm{kHz}, 1.05 \mathrm{kHz}, \ldots$ ) in this ray tracing model. The SPL at each octave band is calculated by the energy sum of each discrete frequency result in that band, but it could be also affected by a slight difference of the frequency step to be chosen.

In contrast, for the case of the measurement result, the peaks and troughs of the sound pressure in space are considered to be less clear due to the effect of diffusion or diffraction in high frequency. Therefore, the measured SPL is probably more robust to the slight difference of the microphone position than the ray tracing analysis result. In this sense, it is believed that the phase interference calculated by the path length difference is no longer realistic in high frequencies. As a result, the octave $1 \mathrm{kHz}$ and $2 \mathrm{kHz}$ results with the phase consideration in Figure 7 show discrepant tendency from the measurement in terms of the magnitude relationship between M3 and M4.

\subsection{Analysis results with seats and tables}

Next, regarding the case after seats and tables were installed, the sound distribution in the longitudinal direction was also analysed and compared with that of the measurement as shown in Figure 8. In addition to the microphone position shown in Figure 6 (M2, M3, M4), microphones at a seat position at the height of $1.2 \mathrm{~m}$ from the floor (M12, M13) were added as the evaluation position. As can be seen from Figure 8, the analysis results show that the SPL of M2 (at the aisle position) is bigger than that of M12 (at the seat position), and the SPL of M3 (aisle position) is bigger than that of M13 (seat position). This tendency is consistent with the measurement results.

However, when comparing the SPL of M12 and M3 and the SPL of M13 and M4, the tendency of the analysis results is different from those of the measurement. The measurement results show that M12 is bigger than M3, and M13 is bigger than M4 in each frequency band. This can be interpreted in that the SPL at the seat position closer to the speaker source (M12, M13) is bigger than the SPL at the aisle position farther from the source (M3, M4). On the contrary, the analysis results show the opposite tendency that M3 is bigger than M12, and M4 is bigger than M13. The reason for this difference may be attributable to the fact that the ray tracing method can consider only the first order diffraction, and the higher order diffraction is neglected.

In real world phenomena, because the sound exhibits wave characteristics, it can go behind the seats by diffraction, and the diffracted sound can further go behind the next seats by higher order diffraction. In this way, the sound can travel in the longitudinal direction even though there are obstacles such as the seats.

In the analysis by the ray tracing method, on the other hand, because only the first order diffraction can be considered, the sound that is diffracted behind the first seat can no longer travel beyond the second seat. Therefore, in the analysis results, the SPL at the seat position (M12, M13) is lower than the SPL at the aisle position (M3, M4), even though the distance from the source is smaller. This is believed to be because the microphone position of M12 and M13 cannot be reached directly from the source, and only the first order diffracted sound (not the second or higher order) and the reflected sound at the wall can reach it in the ray tracing method. The tendency of this opposite phenomena does not change much when the phase correction is considered by applying the coherent source option, which is shown in Figure 9 by the dotted line. Also, Figure 10 shows more clearly the sound distribution of the analysis results, which is an example at $500 \mathrm{~Hz}$. Analysed SPL at the aisle position is generally larger than that of the other parts of the vehicle interior compared at the same distance from the source speaker. This sound distribution also implies that the direct sound from the source contributes the most in the ray tracing analysis, and the SPL at the seating position is underestimated because the higher order diffraction cannot be considered.

However, when considering the actual interior noise in the running condition, it is rare that the point source is placed on one side of the car end, and the sound from this point source is transmitted to the other end of the car. In 
reality, the main noise sources are placed on both ends of the car (above the bogie), and there are also the external aerodynamic noise sources around the car, and the rolling noise sources under the floor, distributed along the longitudinal direction of the train. Since these noise sources are distributed somewhat evenly in space, it is believed that there is little influence of the higher order diffraction in the actual running condition. As a result, there is a possibility that the interior noise in the running condition can be analysed by the ray tracing method with a sufficient degree of accuracy for practical use. Therefore, interior noise analysis in the running condition is discussed in the next chapter.
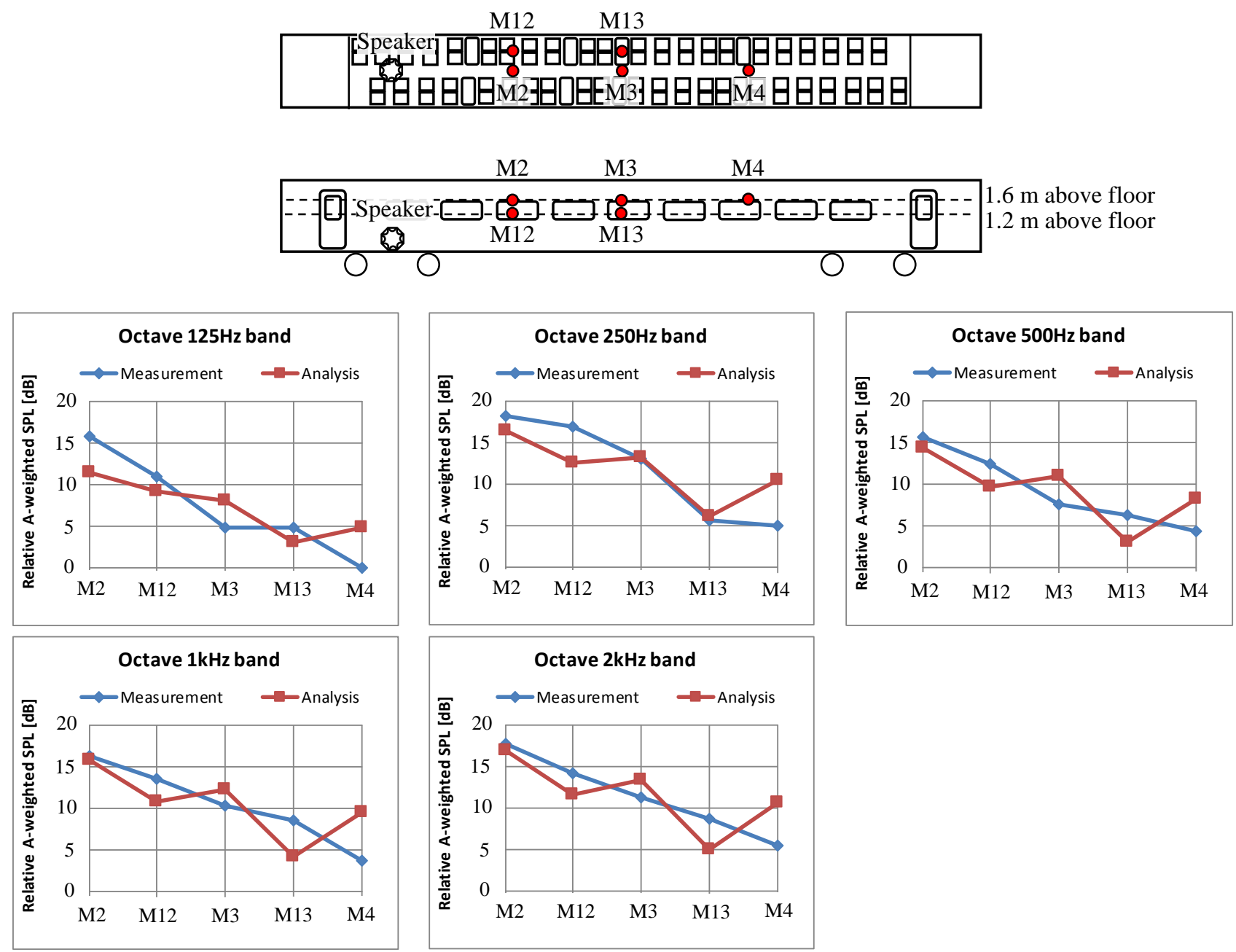

Fig. 8 Analysis results of noise distribution with seats and tables 

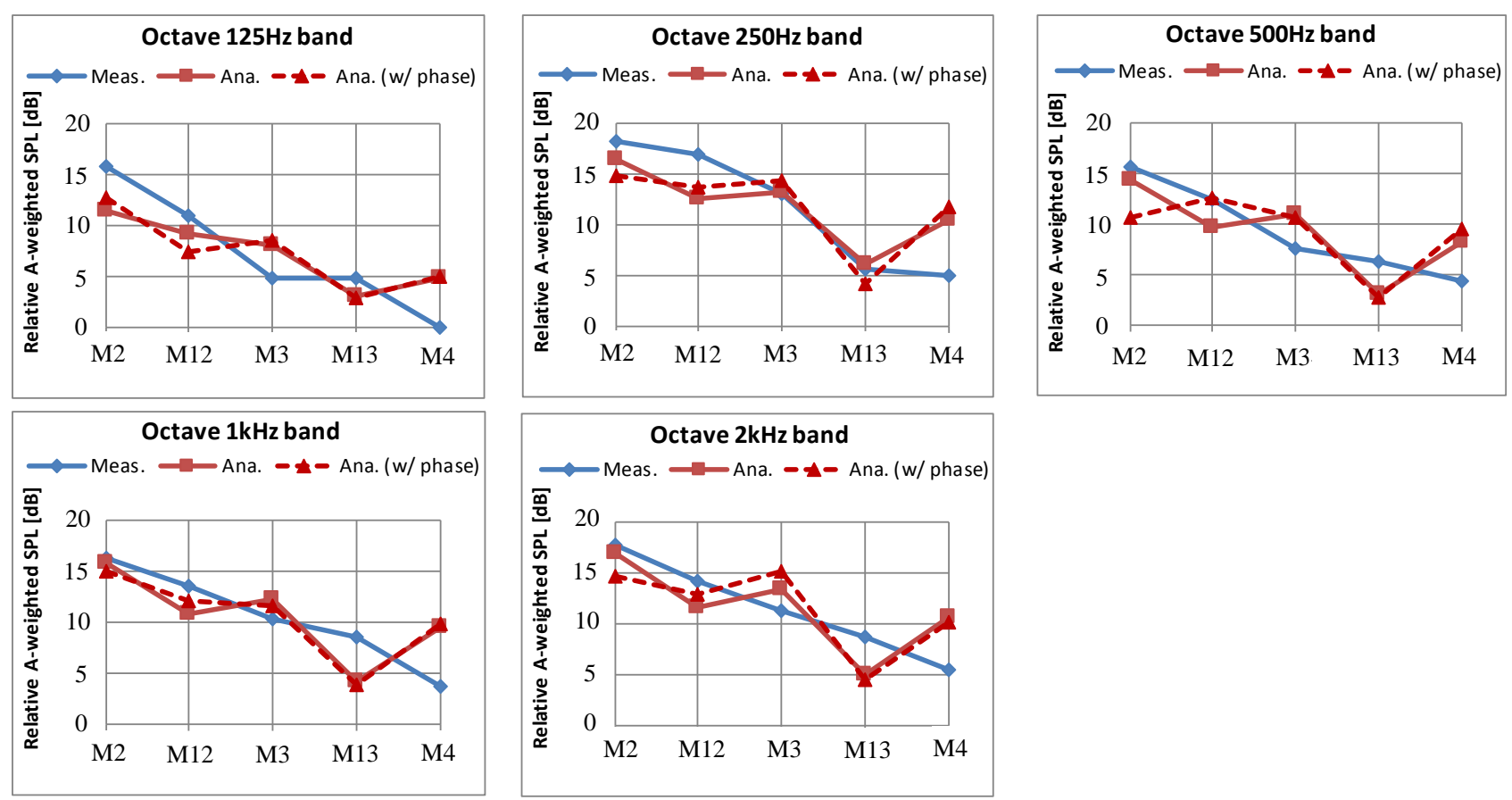

Fig. 9 Analysis results with phase correction (coherent source option)

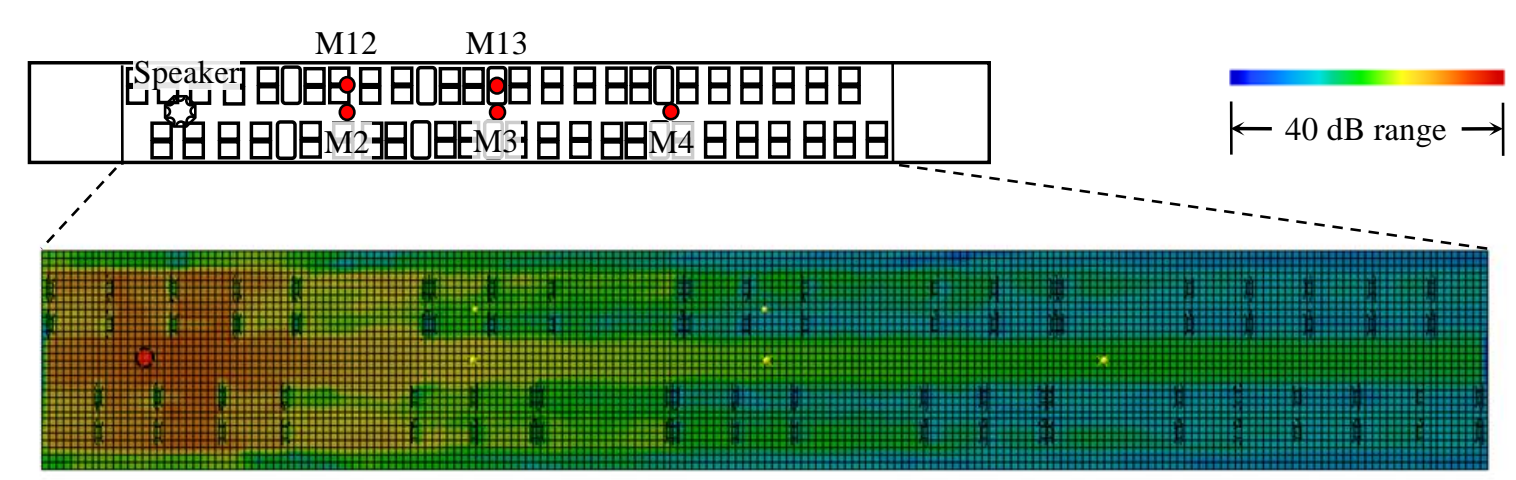

Fig. 10 Example of analysed interior noise distribution at $1.2 \mathrm{~m}$ above floor in $500 \mathrm{~Hz}$ band

\section{Interior noise analysis in the running condition by using the measured vibration of interior panels 4.1 Allocation of input sources}

In order to conduct the interior noise analysis in the running condition, it is assumed that the vibration-induced radiated sounds from the interior surface of the vehicle (such as interior panels, floors, and windows) are dominant as input sources at the maximum speed (i.e. outlet noise from the components like HVAC, etc. can be neglected). In this study, the radiated sound power levels calculated from the measured vibration data of the interior surfaces will be used as input sources. When the surface having an area of $F\left[\mathrm{~m}^{2}\right]$ is vibrating at the velocity of $v[\mathrm{~m} / \mathrm{s}]$, the radiated sound power $W[\mathrm{~W}]$ can be expressed as Equation (3), where $\sigma$ is the radiation efficiency of the surface, $\rho\left[\mathrm{kg} / \mathrm{m}^{3}\right]$ is the density of the air, and $c[\mathrm{~m} / \mathrm{s}]$ is the speed of the sound.

$$
W=\sigma \rho c v^{2}
$$

Here, radiation efficiency $\sigma$ depends on the size of the vibrating surface and the wave length of the sound. In high frequencies in which the sound wave length is shorter than the vibrating surface, $\sigma$ can be assumed as 1 . However, in low frequencies in which the sound wave length is longer than the vibrating surface, $\sigma$ is smaller than 1 , and it is difficult to estimate $\sigma$ with good accuracy. Although there are theories proposed (Leppington et al., 1982), (Xie et al., 2005 ) to estimate the radiation efficiency $\sigma$, the value of $\sigma$ is highly affected by whether the panel is acoustically excited or mechanically excited, as well as by the structural modes and/or the boundary conditions of the panel.

Therefore in this paper, the radiation efficiency $\sigma$ was estimated from the measurement data. Although $\sigma$ may vary 
from surface to surface depending on the structure of each interior panel, in order to simplify the problem it was assumed that $\sigma$ is the same value over the whole surface of the vehicle interior. Then, $\sigma$ was identified so that the calculated interior noise level matches the measured noise level. This identification of $\sigma$ was conducted with the furnished vehicle condition (with seats and tables installed). By using this $\sigma$ for the calculation of the unfurnished vehicle condition (without seats and tables), and by comparing the simulated and measured interior noise level, it is possible to validate the analysis method that is presented here.

Regarding the allocation of the input sources, 9 cross sections from A to I as shown in Figure 11 were considered. At each cross section, 12 sources labelled as V1L, V2L, etc. were defined on each of the interior surfaces of the analysis model. This totals to 108 sources on all surfaces, and these sources were defined as point sources of which acoustic radiation power was estimated from the measured vibration data of the actual vehicle in the running condition.

More specifically, the vibration velocity level of each interior panel and floor was measured by attaching accelerometers on the position where it is labelled as V1L, V2L, etc. in Figure 11. This was mainly measured at the cross section of both car ends above the bogie, namely the cross section A and I. Some of the vibration levels at the position in the middle of the car were also measured but were limited. Therefore, un-measured vibration levels at the cross section from $\mathrm{B}$ to $\mathrm{H}$ were estimated on the basis of the assumption that the vibration decay rate in $\mathrm{dB} / \mathrm{m}$ along the longitudinal direction of the car is the same at each sensor position. Also, accelerometers on the symmetry position such as V1L compared to V1R were also omitted to reduce the number of sensors, so the same vibration level was assumed at the un-measured symmetry position. Finally, the radiation efficiency $\sigma$ and the un-measured vibration distribution from the cross section $\mathrm{B}$ to $\mathrm{H}$ were defined so that the analysis results of the interior noise match the measured SPL at the several microphone positions inside the vehicle. Figure 12 shows the measurement position of the interior noise for the running test with the furnished vehicle condition. S1 to S4 are at the aisle position, which is $1.6 \mathrm{~m}$ above the floor, whereas S5 to S8 are at the seat position at the height of $1.2 \mathrm{~m}$ above the floor.

The detailed procedure for identifying the radiation efficiency $\sigma$ and the vibration distribution at the cross section B to $\mathrm{H}$ is as follows. Firstly, the radiation efficiency $\sigma$ was tentatively defined as 1 . The vibration levels at the un-measured positions in the cross section $\mathrm{B}$ to $\mathrm{H}$ were defined by interpolating the vibration levels in the cross section $\mathrm{A}$ and $\mathrm{I}$ and by using some of the measured vibration data in cross section B to $\mathrm{H}$. The radiated acoustic sound power level from each input position was calculated by Equation (3). The interior noise was analysed using these sound power levels as input sources. Secondly, the radiation efficiency $\sigma$ was adjusted so that the analysed interior noise at S1 and S4 (above the bogie) best matches the measured data. Here, it was assumed that the value of $\sigma$ is the same over the whole interior surface of the vehicle. Finally, the vibration levels at the un-measured positions in the cross section B to $\mathrm{H}$ were adjusted so that the analysed interior noise at S2, S3, and S5-S8 matches the measured data well. Here, it was assumed that the vibration level decreases as the location goes far from the cross section A or I (above the bogie) toward the cross section $\mathrm{E}$ (centre of the car). The estimated radiation efficiency $\sigma$ by this procedure is shown in Figure 13 . In the next section, analysis results of the interior noise by using the input power estimated by this method will be discussed. For the analysis in the running condition, the coherent source option was disabled so that the effect of phase interference is not to be taken into account. 

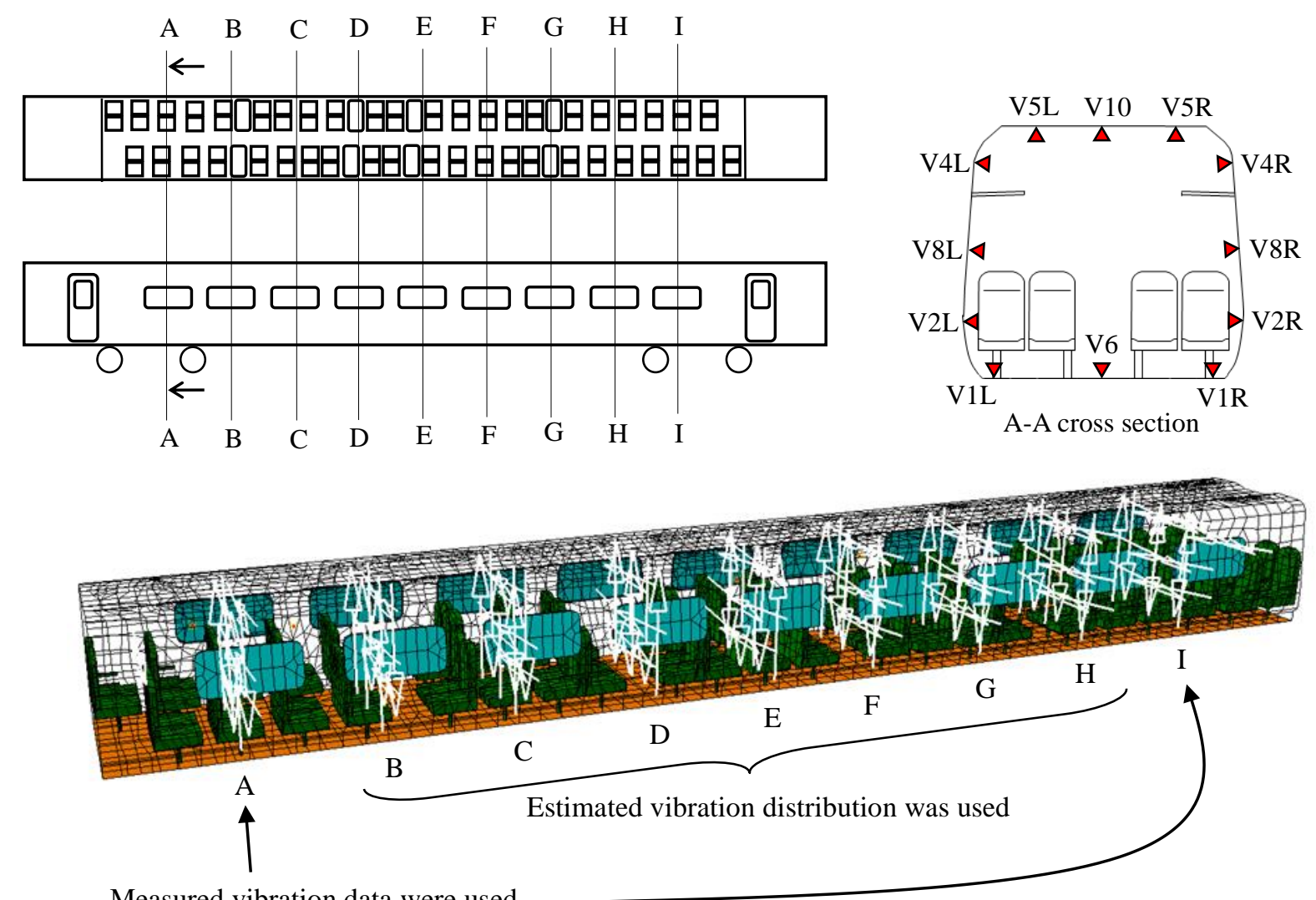

Measured vibration data were used

Fig. 11 Input position of sources on analysis model
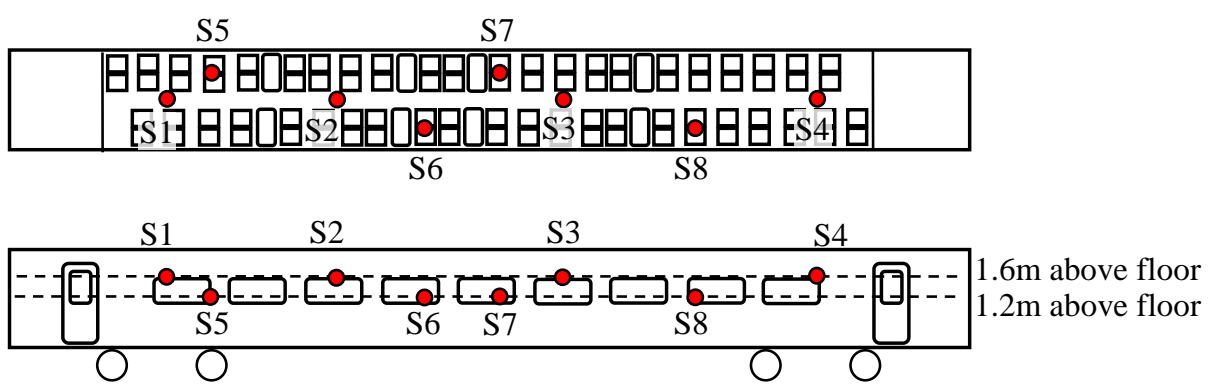

Fig. 12 Measurement position of interior noise

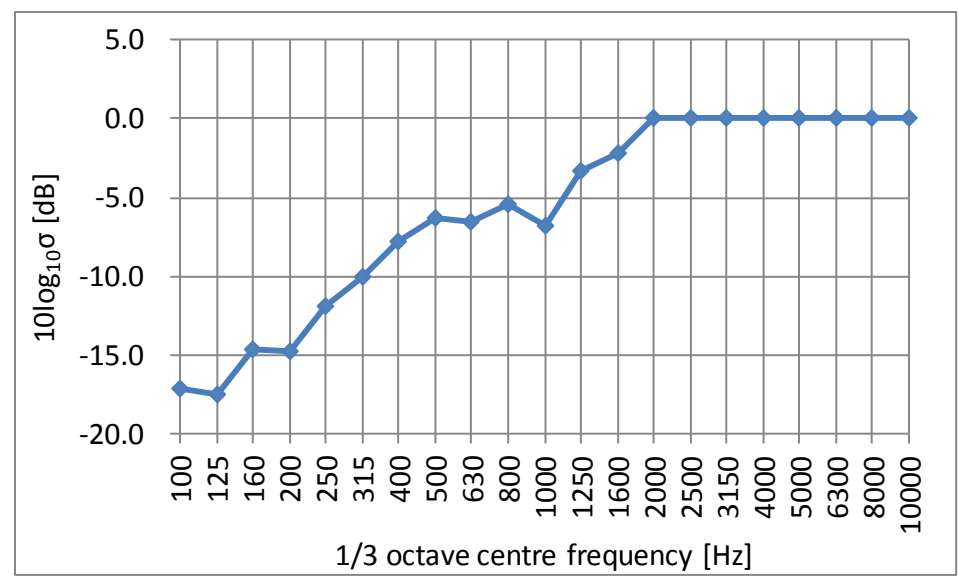

Fig. 13 Estimated radiation efficiency 


\subsection{Validity check of input sources applied to the model}

In order to check the validity of the input sources applied to the simulation model, analysed interior noise was compared with the measurement in the furnished condition (with seats and tables). As explained in the previous section, the input sources were adjusted so that the analysis results in this condition best match the measurement. Figure 14 shows the comparison of analysed and measured noise at S1, S3, S5, and S7 in octave frequency spectra. The microphone position is as shown in Figure 12. S1 and S3 are at the aisle position above the bogie and the centre of the car, respectively, whereas S5 and S7 are at the seating position which is close to S1 and S3, respectively. Although there are deviations to some extent from the measured results at some of the microphone locations in some frequency bands, the analysis results shown in Figure 14 are generally showing a similar trend with the measurement. Thus, the validity of the input sources applied to the analysis model was confirmed. Those deviations of the analysis results may come from the difference of the input power applied to the middle of the car (cross section B to H in Figure 11) because very few accelerometers could be attached in this region because of the limitation of the number of the sensors, and many un-measured vibrations in cross section $\mathrm{B}$ to $\mathrm{H}$ were estimated by interpolating the few measured data in this region and the symmetrically measured half data in cross section A and I (vibration in the symmetry position was omitted). This deviation could be improved by adjusting the input power applied to the simulation model at the un-measured position or by measuring more data on the un-measured position, but it is not the essential purpose of this validation to accurately identify the input sources applied to the model, so it is not pursued here.

\subsection{Analysis results of interior noise change by the effect of seats and tables}

In order to verify the accuracy of the analysis method, interior noise without seats and tables was also analysed, and the interior noise change by the installation of seats and tables was compared to the measured data from the running test. Figure 15 shows the comparison between analysis and measurement. Both analysed and measured results show a similar trend in which the SPL at each microphone position is decreased by the installation of the seats and tables. The results at S2 and S3 show a relatively large difference between analysis and measurement at some frequency bands, which is due to the imperfect identification of the input sources applied to the model as explained above, but the reduction effect by the seats seems to have a similar level between analysis and measurement. Then, the noise reduction effects by the seats were compared as shown in Figure 16. As can be seen from Figure 16, the analysed noise reduction effects by the seats matched those of the measured results within a $2 \mathrm{~dB}$ difference. Therefore, the validity of the presented analysis method was confirmed.

In this study, measured vibration data was used to estimate the input source power from the interior surfaces to achieve enough accuracy of the ray tracing analysis. However, we are also developing a technology to analyse the vibration of interior surfaces based on SEA method (Yoshizawa et al., 2013). When it becomes possible to estimate the vibration distribution of interior surfaces in running condition with enough accuracy, those estimated vibration can be used as inputs to the ray tracing model. In that case, it will be possible to conduct full interior noise analysis without the measured vibration data in running condition. 

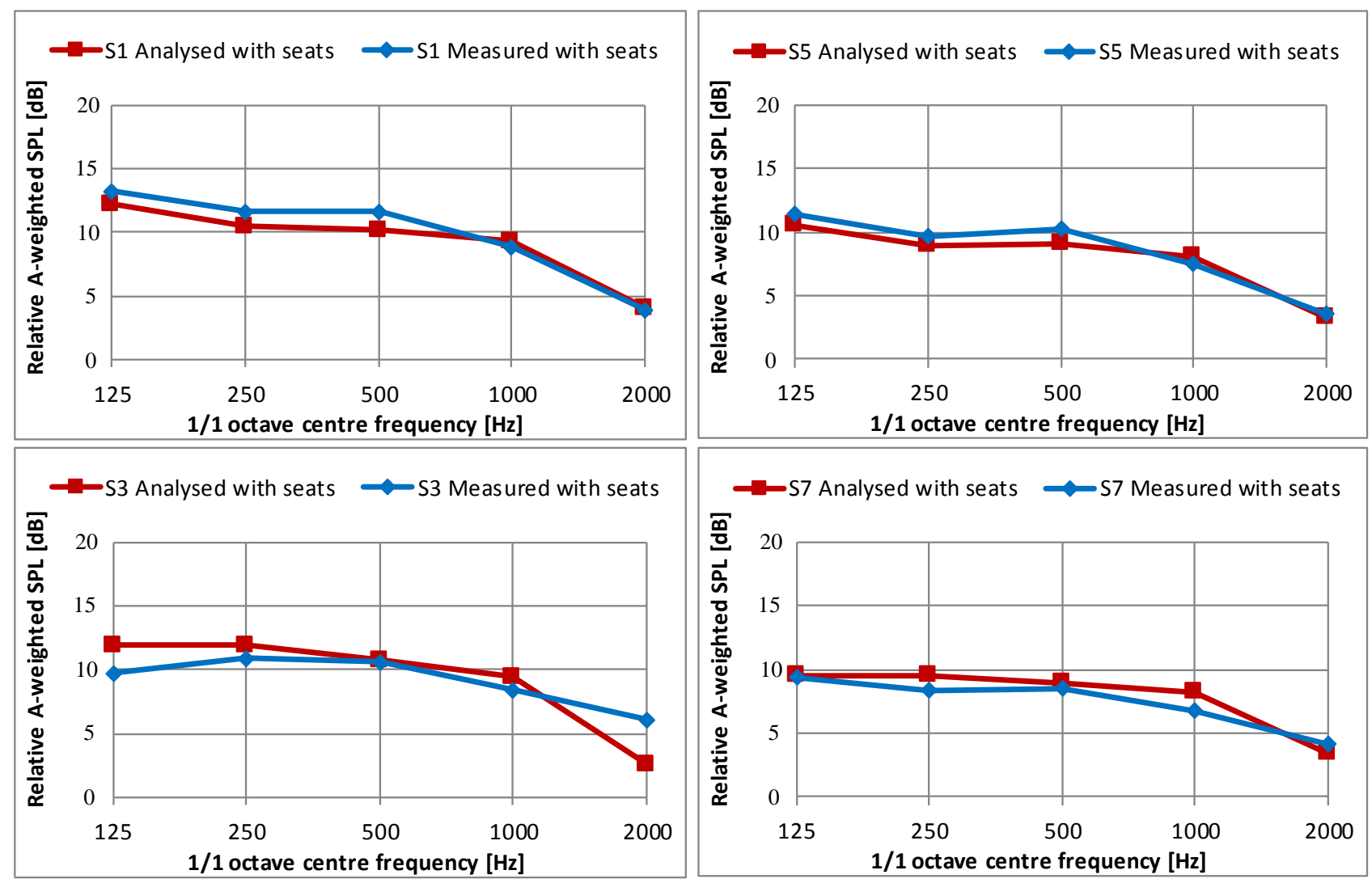

Fig. 14 Analysis results with seats and tables for the validity check of applied input sources
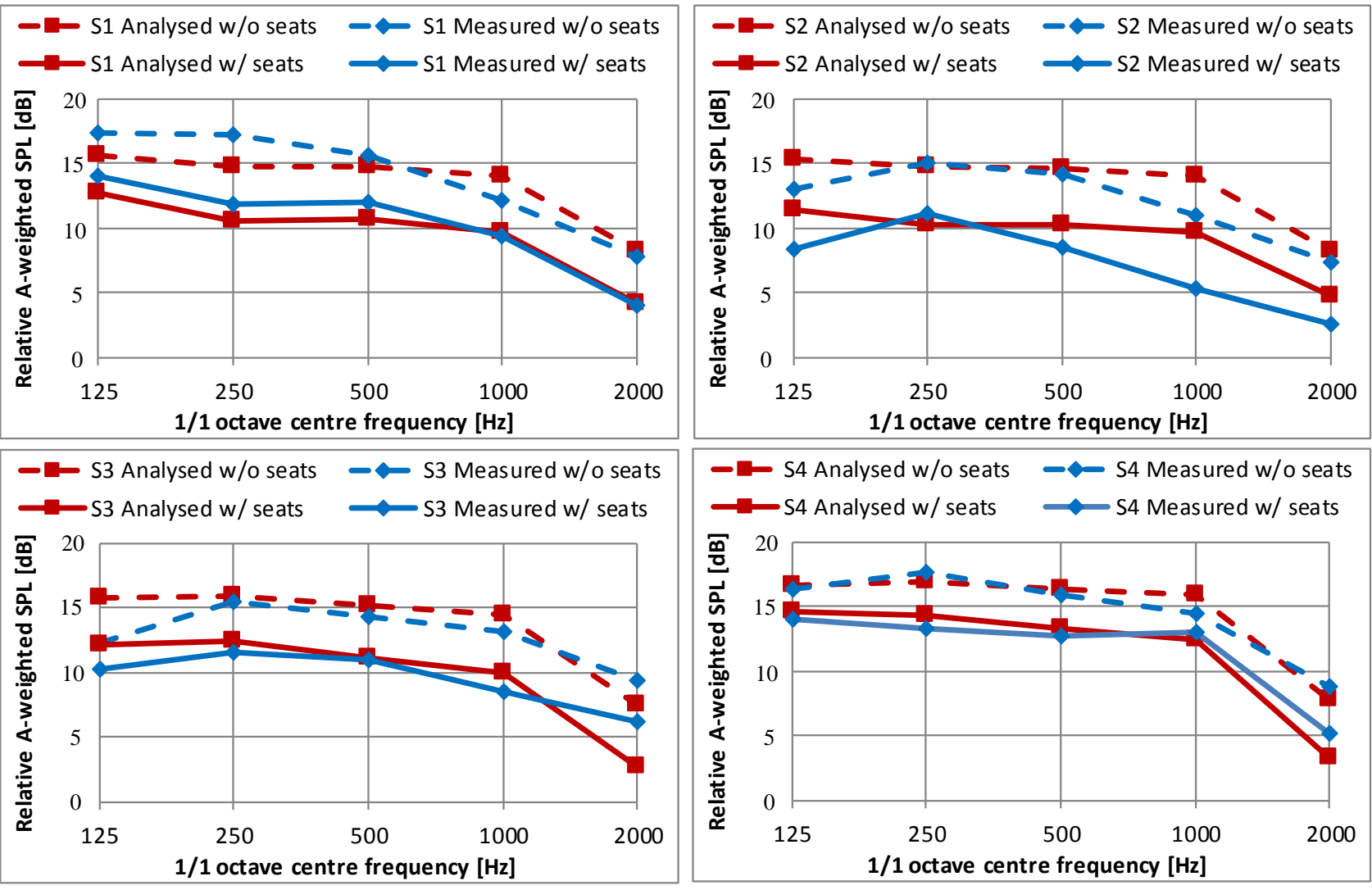

Fig. 15 Analysis and measurement comparison of interior noise with and without seats 

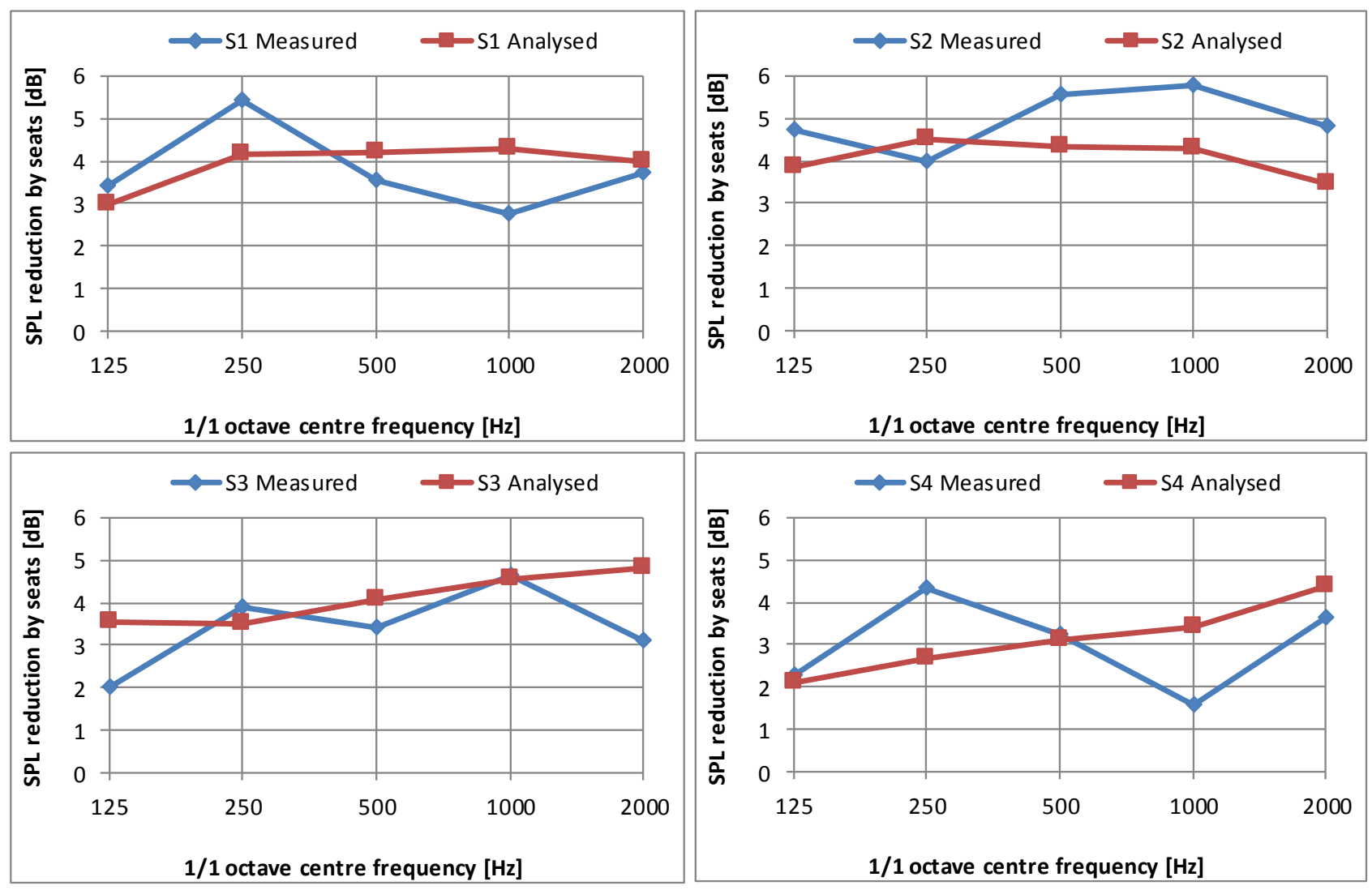

Fig. 16 Analysis and measurement comparison of noise reduction effect by seats

\section{Conclusion}

We have studied the analysis method of interior noise in railway cars by means of the ray tracing method. The analysed sound distribution in the longitudinal direction by using a point source as input, that is placed at one end of the car, did not always match the measurement results obtained by a loud speaker test. This is believed to be because the wave character of the sound cannot be strictly simulated by the ray tracing method. However, in regards to the analysis for the running condition, input sources are generally distributed inside the whole vehicle. Therefore, multiple sources were defined on the interior surface of the car, and the amplitude of each source was estimated from the measured vibration of the interior panels. As a result, it was found that the interior noise changes by the installation of seats and tables agreed well with those of the measurement. Therefore, it was confirmed that the interior noise can be analysed with practically enough accuracy by the method presented in this study.

\section{References}

Architectural Institute of Japan ed., Beginners' programming guide to numerical acoustic simulation (2012), Chapter 7 : Ray tracing method, pp. 166-207, Corona Publishing (in Japanese).

Forssen, J., Tober, S., Corakci, A. C., Frid, A., and Kropp, W., Modelling the interior sound field of a railway vehicle using statistical energy analysis, Applied Acoustics, Volume 73, Issue 4 (2012), pp. 307-311.

Gabet, P., Drobecq, N., Noe, N., and Jean, P., Prediction of Acoustic Comfort of a Trainset using Ray-Tracing Technology, Proceedings of Confort Automobile et Ferroviaire (2008).

Leppington, F. G., Broadbent, E. G., and Heron, K. H., The acoustic radiation efficiency of rectangular panels, Proceedings of the Royal Society London A 382 (1982), pp. 245-271.

LMS International, RAYNOISE Revision 3.1 User's Manual, 1998.

Muto, D., Takano, Y., Takeichi, M., Makino, K., and Shigeyama, M., Statistical Energy Analysis of Interior Noise in a High-speed Train, Proceedings of Inter noise 2003, N917. 
Poisson, F., Loizeau, T., Bouvet, S., and Vincent, N., Transfer Path Analysis Within a TGV Duplex Coach, Proceedings of ICSV 16 (2009).

Savioja, L. and Svensson, U. P., Overview of geometrical room acoustic modeling techniques, Journal of the Acoustical Society of America, Vol. 138, Issue 2 (2015), pp. 708-730.

Yoshizawa, T., Takano, Y., Mochida, T., Sebata, M., and Makino, K., Interior noise prediction of a rolling stock using statistical energy analysis method, Proceedings of Inter noise 2013, No. 634.

Xie, G., Thompson, D. J., and Jones, C. J. C., The radiation efficiency of baffled plates and strips, Journal of Sound and Vibration, Vol. 280 (2005), pp. 181-209. 